OPEN ACCESS

Check for updates

\title{
Association of ultra-processed food intake with risk of inflammatory bowel disease: prospective cohort study
}

\author{
Neeraj Narula, ${ }^{1,2}$ Emily C L Wong, ${ }^{1}$ Mahshid Dehghan, ${ }^{2}$ Andrew Mente, ${ }^{2}$ Sumathy Rangarajan, ${ }^{2}$ \\ Fernando Lanas, ${ }^{3}$ Patricio Lopez-Jaramillo, ${ }^{4}$ Priyanka Rohatgi, ${ }^{5}$ P V M Lakshmi, ${ }^{6}$ \\ Ravi Prasad Varma, ${ }^{7}$ Andres Orlandini, ${ }^{8}$ Alvaro Avezum, ${ }^{9}$ Andreas Wielgosz, ${ }^{10}$ Paul Poirier, ${ }^{11}$ \\ Majid A Almadi, ${ }^{12}$ Yuksel Altuntas, ${ }^{13}$ Kien Keat Ng, ${ }^{14}$ Jephat Chifamba, ${ }^{15}$ Karen Yeates, ${ }^{16}$ \\ Thandi Puoane, ${ }^{17}$ Rasha Khatib, ${ }^{18}$ Rita Yusuf, ${ }^{19,20}$ Kristina Bengtsson Boström, ${ }^{21}$ \\ Katarzyna Zatonska, ${ }^{22}$ Romaina Iqbal, ${ }^{23}$ Liu Weida, ${ }^{24}$ Zhu Yibing, ${ }^{24}$ Li Sidong, ${ }^{24}$ \\ Antonio Dans, ${ }^{25}$ Afzalhussein Yusufali, ${ }^{26}$ Noushin Mohammadifard, ${ }^{27}$ John K Marshall, ${ }^{1}$ \\ Paul Moayyedi, ${ }^{12}$ Walter Reinisch, ${ }^{28}$ Salim Yusuf ${ }^{2}$
}

For numbered affiliations see end of the article

Correspondence to: N Narula neeraj.narula@medportal.ca (or@narulaneeraj on Twitter ORCID 0000-0002-1536-8436) Additional material is published online only. To view please visit the journal online.

Cite this as: BMJ 2021;374:n1554 http://dx.doi.org/10.1136/bmj.n1554 Accepted: 14 June 2021

\section{ABSTRACT}

OBJECTIVE

To evaluate the relation between intake of ultraprocessed food and risk of inflammatory bowel disease (IBD).

DESIGN

Prospective cohort study.

SETTING

21 low, middle, and high income countries across seven geographical regions (Europe and North America, South America, Africa, Middle East, south Asia, South East Asia, and China).

\section{PARTICIPANTS}

116087 adults aged 35-70 years with at least one cycle of follow-up and complete baseline food frequency questionnaire (FFQ) data (country specific validated FFQs were used to document baseline dietary intake). Participants were followed prospectively at least every three years.

\section{MAIN OUTCOME MEASURES}

The main outcome was development of IBD, including Crohn's disease or ulcerative colitis. Associations between ultra-processed food intake and risk of IBD were assessed using Cox proportional hazard multivariable models. Results are presented as hazard ratios with $95 \%$ confidence intervals.

RESULTS

Participants were enrolled in the study between 2003 and 2016. During the median follow-up of 9.7 years (interquartile range 8.9-11.2 years), 467 participants

\section{WHAT IS ALREADY KNOWN ON THIS TOPIC}

Inflammatory bowel disease (IBD) is more common in industrialised nations It is hypothesised that environmental factors such as diet might influence the risk of IBD

Many dietary risk factors have been investigated for an association with IBD, but data for an association between ultra-processed food (which contain additives and preservatives) intake and IBD are limited

\section{WHAT THIS STUDY ADDS}

A higher intake of ultra-processed food was associated with higher risk of IBD Individual food categories (meats, dairy, starches, fruit, and vegetables) were not associated with risk of IBD, suggesting that risk might be related to the food itself rather than the way it is processed or ultra-processed developed incident IBD (90 with Crohn's disease and 377 with ulcerative colitis). After adjustment for potential confounding factors, higher intake of ultra-processed food was associated with a higher risk of incident IBD (hazard ratio 1.82, 95\% confidence interval 1.22 to 2.72 for $\geq 5$ servings/day and 1.67 , 1.18 to 2.37 for $1-4$ servings/day compared with $<1$ serving/day, $\mathrm{P}=0.006$ for trend). Different subgroups of ultra-processed food, including soft drinks, refined sweetened foods, salty snacks, and processed meat, each were associated with higher hazard ratios for IBD. Results were consistent for Crohn's disease and ulcerative colitis with low heterogeneity. Intakes of white meat, red meat, dairy, starch, and fruit, vegetables, and legumes were not associated with incident IBD.

\section{CONCLUSIONS}

Higher intake of ultra-processed food was positively associated with risk of IBD. Further studies are needed to identify the contributory factors within ultraprocessed foods.

STUDY REGISTRATION

ClinicalTrials.gov NCT03225586.

\section{Introduction}

Inflammatory bowel disease (IBD), comprised of Crohn's disease and ulcerative colitis, is a chronic inflammatory disorder of the gastrointestinal tract. The pathophysiology of IBD is thought to be related to activation of the intestinal mucosal immune system in response to dysbiosis of the gastrointestinal tract in genetically susceptible people. ${ }^{1}$ Diet alters the microbiome $^{2}$ and modifies the intestinal immune response and so could play a role in the pathogenesis of IBD. ${ }^{3}$ The incidence of IBD has increased in several countries where both diseases were previously uncommon. ${ }^{4}$ This increase has paralleled the adoption of a western diet in these countries. Dietary changes in such countries, including increased intake of refined sugars and dietary fats such as n-6 polyunsaturated fatty acids and decreased intake of fibre, have been suggested as potential risk factors for the development of IBD. ${ }^{5-7}$

Specific data linking dietary factors with IBD in human populations have been limited and conflicting. ${ }^{8-10}$ Most previous studies of diet and IBD 
have used retrospective or case-control designs. ${ }^{9}{ }^{10}$ The few prospective studies that have examined dietary risk factors have been limited by small numbers of participants, lack of adjustment for potential confounders, or use of homogenous populations confined to individual countries or specific regions of countries. ${ }^{6711-15}$

A recent systematic review synthesised all studies that evaluated dietary intake and risk of IBD. ${ }^{10}$ It was evident from the findings that many associations have been examined repeatedly, including the association of different dietary fats, carbohydrates, proteins, fruit, vegetables, fibre, and dairy with IBD. ${ }^{10}$ Meats have been assessed as a whole, with some, but not all, studies suggesting an increased odds for the development of IBD with higher meat intake. ${ }^{1016}$ The systematic review also found high intake of total fats, polyunsaturated fatty acids, and omega- 6 fatty acids to be associated with increased risk of IBD. High fibre and fruit intake might decrease the risk of Crohn's disease, and high vegetable intake was associated with a decreased risk of ulcerative colitis. ${ }^{17} 18$

Recent attention has focused on the non-nutritional components of diet and potential associated risks. Processed foods often include many non-natural ingredients and additives such as artificial flavours, sugars, stabilisers, emulsifiers, and preservatives. Detergents and emulsifiers that are added to foods might have a detrimental effect on the gut barrier. Carboxymethylcellulose has been shown to increase bacterial adherence to intestinal epithelium and might lead to bacterial overgrowth and infiltration of bacteria into the spaces between intestinal villi. ${ }^{19}$ Polysorbate 80 , an emulsifier commonly used in processed foods, increases translocation of bacteria such as Escherichia coli across M cells and Peyer's patches in people with Crohn's disease. ${ }^{20}$ Associations have been reported between diets high in processed foods and development of diseases such as cancer and cardiovascular disease. $^{2122}$ One recent study investigated whether ultra-processed foods were associated with risk of IBD and did not find any association, but this study was limited by few patients with IBD (75 among 105832 participants). ${ }^{15}$

Using information from the Prospective Urban Rural Epidemiology (PURE) cohort, we describe the association between ultra-processed food intake and risk of developing IBD. ${ }^{24-26}$

\section{Methods}

The design and methods of the PURE study have been described and published previously. ${ }^{23-25}$ The first and second phases of the study took place between 1 January 2003 and 31 December 2016 and included 136384 adults aged 35-70 years who had dietary information assessed. Participants were enrolled from 21 countries: Argentina, Bangladesh, Brazil, Canada, Chile, China, Colombia, India, Iran, Malaysia, Palestine, Pakistan, Philippines, Poland, South Africa, Saudi Arabia, Sweden, Tanzania, Turkey, United Arab Emirates, and Zimbabwe. The sampling and recruitment strategies from PURE have been published previously (see supplementary appendix 1). ${ }^{26}$ Data were collected at the community, household, and individual level using standardised questionnaires. Standard case report forms were used to record new diagnoses of Crohn's disease and ulcerative colitis during the follow-up period. For the current analysis, the data include all outcome events up to 5 July 2019. The Population Health Research Institute and McMaster University and Hamilton Health Sciences in Hamilton, Ontario, Canada coordinated the study.

\section{Procedures}

For each participant in PURE, usual food intake was assessed at baseline using country specific validated food frequency questionnaires (FFQ). Validated FFQs were developed for countries where such questionnaires had not been available previously (supplementary table 1). Participants received a list of food items and were asked to input their frequency for intake of each item in the past year. To compute daily food and nutrient intakes, participant's answers were converted to daily intake and multiplied by United States Department of Agriculture (USDA) serving size. Ultra-processed food included all types of packaged and formulated foods and beverages that contain food additives, artificial flavourings, colours, or other chemical ingredients. Included in this group were processed meat, cold breakfast cereal, various types of sauce, soft drinks, refined sweetened foods (eg, candy, chocolate, jam, jelly, brownies, pudding), chips, ice cream, commercially prepared pastries, biscuits, and fruit drinks. To make the unit of consumption consistent between countries, we used daily serving intake.

\section{Outcomes}

The primary outcome was the development of IBD after completion of the baseline questionnaire. Other outcomes of interest were development of Crohn's disease and ulcerative colitis individually by dietary risk factors that had statistically significant associations with the development of IBD overall. Although data collection for PURE started in 2003, an amendment was implemented in 2014 to record diagnoses of Crohn's disease and ulcerative colitis. Participants were asked at every follow-up questionnaire whether they had a diagnosis of Crohn's disease or ulcerative colitis. They were asked when the condition was diagnosed and those with prevalent diagnoses of IBD (diagnosis before baseline questionnaire) were excluded. Participants were also excluded if their baseline FFQ was completed less than one year from the time of the reported IBD diagnosis. Participants self-reported the diagnosis. A validation exercise was conducted to validate $20 \%$ of the diagnoses using a sample of the population where medical records could be provided for review by the authors.

\section{Statistical analysis}

Continuous variables are expressed as means with standard deviation, and categorical variables are 
expressed as percentages. Education was categorised as college, university, or trade (age >11 years), secondary or high school (age 7-11 years), or none or primary school (age $<6$ years). Alcohol was categorised as <1 serving/month (eg, per serving: beer $14 \mathrm{~g}$, wine $16 \mathrm{~g}$, vodka, rum, spirits $10 \mathrm{~g}$, liqueur $27 \mathrm{~g}$ ), 1 serving/ month to $1 /$ week, or $>1$ serving/week. Geographical region was treated as binary variable of North America and Europe or rest of the world because the prevalence of IBD is typically highest in North America and Europe and lower in other parts of the world. Other binary variables included smoking status (current versus no smoking) and location (rural versus urban). Other categorical variables included body mass index, classified as obese, overweight, or normal, and physical activity, classified as low (<600 metabolic equivalent of task (MET) min/week), moderate (6003000 MET min/week), or high (>3000 MET min/week). Continuous variables included age, daily total energy (in kcal), and waist to hip ratio.

For the overall analysis of total ultra-processed food, participants were grouped into categories according to servings as $<1$ serving/day, $1-4$ servings/day, or $\geq 5$ servings/day. The lowest intake group was always used as the reference group. In a sensitivity analysis, we also grouped participants based on grams of intake of ultraprocessed food into $<50 \mathrm{~g} /$ day, 50-99 g/day, and $\geq 100 \mathrm{~g} /$ day. We also assessed the association using categories of ultra-processed foods individually. Processed meat was categorised into <1 serving/week, 1-6 servings/ week, or $\geq 7$ servings/week. Soft drink intake was categorised into $<0.5$ serving/week, 0.5 to $<3$ servings/ week, or $\geq 3$ servings/week. Refined sweetened foodsthose high in refined sugars (eg, cake, cookies)-and intake was categorised as none, 1-99 g/day, or $\geq 100 \mathrm{~g} /$ day. Salty foods and snacks (eg, crackers, potato chips, nachos, popcorn) were categorised into $<50 \mathrm{~g} /$ day, 50$99 \mathrm{~g} /$ day, and $\geq 100 \mathrm{~g} /$ day. An additional analysis of urinary sodium was conducted, as a surrogate of dietary sodium, to determine whether dietary sodium could potentially be implicated should ultra-processed foods be associated with risk of IBD. The Kawasaki formula was used to estimate urinary sodium excretion. ${ }^{27}$ Based on a previous study assessing urinary sodium, categories chosen were <2.5 g/day, 2.5-3.4 g/day, and $\geq 3.5 \mathrm{~g} /$ day. $^{27}$ A sensitivity analysis was planned to exclude China because of its significantly higher observed sodium intake compared with the rest of the world and relatively low event rates for IBD. ${ }^{27}$

Exploratory analyses were planned with other dietary variables to confirm or refute risk factors that had been reported in the literature as showing associations. Participants were categorised by white meat intake into <1 serving/week, 1-2 servings/week, or $\geq 3$ servings/week. Red meat (unprocessed) was categorised into $<3$ servings/week, 3-6 servings/week, or $\geq 7$ servings/week. Dairy intake was categorised into $<1$ serving/day, 1 to $<2$ servings/day, or $\geq 2$ servings/ day. Starch intake was categorised into $<200 \mathrm{~g} /$ day, 200-399 g/day, and $\geq 400 \mathrm{~g} /$ day. Fruit, vegetables, and legumes were analysed together and classified as $<2$ servings/day, 2-5 servings/day, and $\geq 6$ servings/day and were also analysed separately. Lastly, fried foods were categorised into none, 1 serving/week to $<1$ serving/day, or $\geq 1$ servings/day.

We calculated hazard ratios using multivariable Cox proportional hazard models. Estimates of hazard ratios and 95\% confidence intervals are presented for each dietary risk factor of interest. Results are presented as unadjusted hazard ratios, minimally adjusted hazard ratios for age, sex, and geographical region, and fully adjusted hazard ratios. Covariates included in the fully adjusted model were those known from previous literature to have an association with the development of IBD (eg, smoking) or those found to have an association on univariable analyses $(\mathrm{P}<0.15)$. Covariates for consideration in the fully adjusted multivariable model included age, sex, geographical region, education, alcohol intake, smoking status, physical activity, energy intake, BMI, waist to hip ratio, and urban versus rural location. An additional model was run including adjustment for diet quality as measured by the Alternate Health Eating Index (AHEI). ${ }^{28}$ The Kolmogorov-type Supremum test was used to test the proportional hazards assumptions. ${ }^{29}$ The $\chi^{2}$ test of linear trend was used to compare across categories of food intake. A two sided $\mathrm{P}<0.05$ was considered to be statistically significant. Sensitivity analysis were conducted by using multiple imputation for participants with missing FFQ data to determine whether this had any impact on the results. For separate analyses of Crohn's disease and ulcerative colitis conducted when a dietary risk factor had statistically significant association with development of IBD, we anticipated lower power to achieve statistical significance separately within each subgroup. For comparison of subgroup effects, we assessed heterogeneity by calculating the $\chi^{2}$ and $\mathrm{I}^{2}$ statistics. For the $\chi^{2}$ test, we considered a $\mathrm{P}$ value of $<0.10$ to be statistically significant. $\mathrm{I}^{2}$ values greater than $50 \%$ were considered to indicate substantial heterogeneity. We used heterogeneity of the subgroup analyses to determine whether the association was predominantly due to effect from one subgroup. Data were analysed using Stata/IC 15.

\section{Patient and public involvement}

No patients were involved in setting the research question or outcome measures, or in the design and implementation of this study.

\section{Results}

Between 1 January 2003 and 31 December 2016, a total of 153220 participants completed the FFQ. Of these participants, 126662 had a plausible energy intake (500-5000 kcal/day; $1 \mathrm{kcal}=4.18 \mathrm{~kJ}$ or 0.00418 MJ) and had at least one cycle of follow-up. Overall, 10625 (8.3\%) were excluded owing to incomplete FFQ data, leaving a total of 116037 participants included in this study.

During a median follow-up of 9.7 years (interquartile range 8.9-11.2 years), 467 participants $(0.4 \%)$ had a 


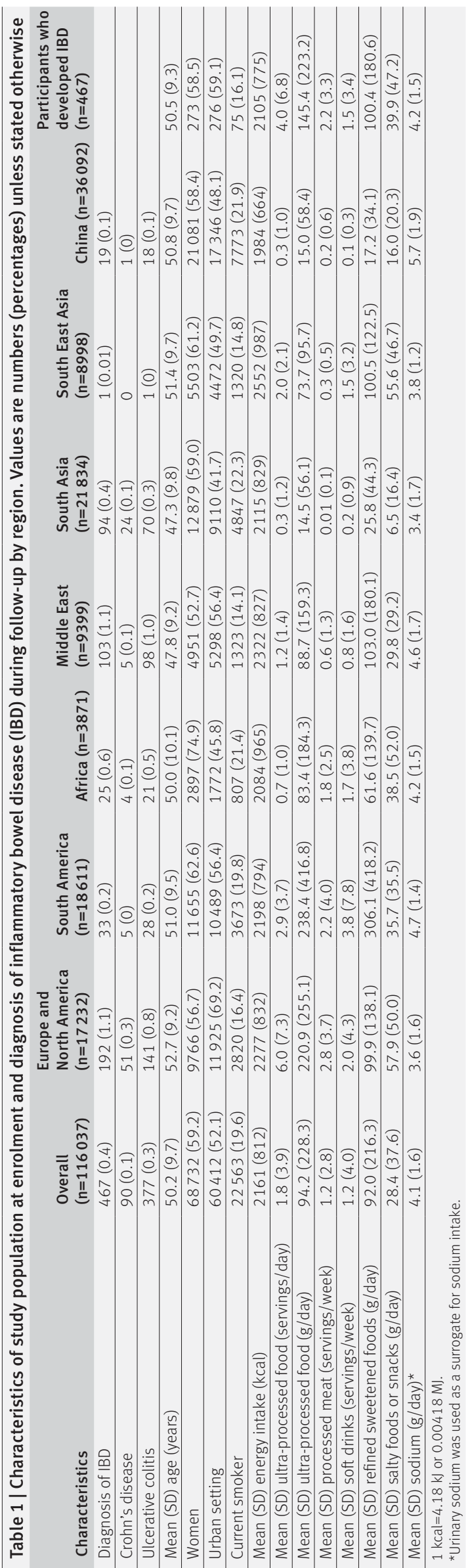

diagnosis of incident IBD (supplementary figure 1): 90 participants with Crohn's disease and 377 with ulcerative colitis. Supplementary appendix 2 provides the results of the validation exercise.

Ultra-processed food intake was higher in North America, Europe, and South America than in other regions (table 1), both in servings and grams of intake daily. Similarly, processed meat and soft drink intakes were highest in these three regions. Consumption of refined sweetened foods was highest in South America, followed by the Middle East and South East Asia. Salty and snack food intake was highest in North America and South East Asia. Sodium intake was highest in China.

Table 2 summarises the results of the univariable associations. Those dietary variables that were nominally significant wereincluded in the multivariable Cox proportional hazard regression models. In addition to age, sex, and geographical region used in the minimally adjusted model, education, alcohol intake, smoking status, BMI, total energy intake, and location were all found to be significantly associated with the development of IBD and were included within the multivariable model. None of the variables in the model violated the proportional hazards assumption $(\mathrm{P}>0.05)$ when tested using the Supremum test for total ultra-processed food intake, individual categories of ultra-processed food intake, urinary sodium, and other food categories, and the outcome of IBD development.

\section{Ultra-processed food intake and risk of IBD}

Table 3 shows the graded association between intake of total ultra-processed foods and risk of IBD. Higher intake of ultra-processed food was associated with a higher risk of incident IBD (hazard ratio 1.82, 95\% confidence interval 1.22 to 2.72 for $\geq 5$ servings/day and $1.67,1.18$ to 2.37 for $1-4$ servings/day compared with $<1$ serving/day, $\mathrm{P}=0.006$ for trend). When further adjusted using the AHEI, findings were similar, as higher risk of IBD was observed for $\geq 5$ servings/day (1.92, 1.28 to 2.90$)$ and $1-4$ servings/day $(1.75,1.23$ to 2.50$)$ compared with $<1$ serving/day $(\mathrm{P}=0.004$ for trend). The results were directionally consistent for Crohn's disease and ulcerative colitis, with no statistical evidence of heterogeneity $\left(\chi^{2} \mathrm{P}=0.60\right.$; $\mathrm{I}^{2}=0 \%$ ). In sensitivity analysis (supplementary table 2), the risk of IBD development in participants with ultra-processed food intake (g/day) was also found to be significantly increased. Higher intake ( $\mathrm{g} /$ day) of ultra-processed food was associated with higher risk of incident $\operatorname{IBD}(1.73,1.23$ to 2.45 for $\geq 100 \mathrm{~g} /$ day and $1.33,0.88$ to 2.01 for $50-100 \mathrm{~g} /$ day compared with $<50 \mathrm{~g} /$ day, $\mathrm{P}=0.007$ for trend). Sensitivity analysis with multiple imputation for missing FFQ data also showed a higher risk of IBD with increased ultra-processed food intake, suggesting that the missing data did not alter the findings (table 3). Subgroup analyses were performed to determine whether participant age was an effect modifier, but similar patterns of increased hazard ratios at higher levels of ultra-processed food intake were observed in participants younger than 


\begin{tabular}{|c|c|c|c|c|}
\hline Predictors & \multicolumn{2}{|l|}{ Univariable } & \multicolumn{2}{|l|}{ Multivariable* } \\
\hline Age & $1.00(0.99$ to 1.01$)$ & 0.51 & 1.01 (0.99 to 1.02$)$ & 0.16 \\
\hline Sex (men) & $1.03(0.86$ to 1.24$)$ & 0.73 & $0.93(0.72$ to 1.20$)$ & 0.59 \\
\hline North America and Europe & $4.04(3.36$ to 4.86$)$ & $<0.001$ & 5.57 (3.97 to 7.81$)$ & $<0.001$ \\
\hline None & 1.00 (ref) & & 1.00 (ref) & \\
\hline Secondary or high school & $1.26(1.01$ to 1.58$)$ & & $1.60(1.13$ to 2.26$)$ & \\
\hline College, university, or trade & 2.24 (1.78 to 2.81$)$ & & 1.57 (1.07 to 2.26$)$ & \\
\hline Alcoholt: & & $<0.001$ & & 0.34 \\
\hline <1 serving/month & 1.00 (ref) & & 1.00 (ref) & \\
\hline Low $(\ltimes 600)$ & 1.00 (ref) & & & \\
\hline Moderate $(600$ to <3000) & $1.28(0.95$ to 1.71$)$ & & & \\
\hline High $(\geq 3000)$ & $1.30(0.98$ to 1.74$)$ & & & \\
\hline Energy (kcal) & $1.00(0.99$ to 1.00$)$ & 0.13 & $1.00(0.99$ to 1.00$)$ & 0.08 \\
\hline Body mass index: & & 0.03 & & 0.78 \\
\hline Normal $(<25)$ & 1.00 (ref) & & 1.00 (ref) & \\
\hline Overweight $(25$ to <30) & 1.09 (0.88 to 1.34$)$ & & 0.91 (0.69 to 1.19) & \\
\hline Obese $(\geq 30)$ & 1.38 (1.09 to 1.75$)$ & & $0.96(0.70$ to 1.32$)$ & \\
\hline Waist to hip ratio & $1.80(0.61$ to 5.28$)$ & 0.28 & & \\
\hline Location: & & 0.002 & & 0.91 \\
\hline
\end{tabular}

50 and 50 or older (supplementary tables 3 and 4). Region specific analyses (supplementary figure 2) were also performed to determine whether the pattern of increased ultra-processed food intake and higher risk of IBD persisted within each of the regions examined, and effect estimates were generally similar, with overlapping confidence intervals and no significant heterogeneity $\left(\chi^{2} \mathrm{P}=0.69 ; \mathrm{I}^{2}=0 \%\right)$.

Supplementary table 5 shows that higher processed meat intake was associated with higher risk of IBD (2.07, 1.14 to 3.76 for $\geq 1$ serving/day and 1.92, 1.242.98 for 1 serving/week to $<1$ serving/day compared with $<1$ serving/week, $\mathrm{P}=0.01$ for trend; supplementary tables 5-8). Increased risk was observed for both Crohn's disease and ulcerative colitis (tests of heterogeneity: $\chi^{2}$ $\left.\mathrm{P}=0.93 ; \mathrm{I}^{2}=0 \%\right)$. A graded risk was also found for soft drinks intake and risk of IBD (supplementary table 6). The highest intake of soft drinks ( $\geq 3$ servings/week) compared with lowest intake $(<0.5$ serving/week) was associated with a higher risk of IBD (1.94, 1.42 to 2.66, $\mathrm{P}<0.001$ for trend), with directionally consistent results observed for Crohn's disease and ulcerative colitis and no statistical evidence of heterogeneity $\left(\chi^{2}\right.$ $\left.\mathrm{P}=0.72 ; \mathrm{I}^{2}=0 \%\right)$.

Consumption of refined sweetened foods was associated with higher risk of IBD (supplementary table $7)$. The highest risk of IBD was observed in participants with $\geq 100 \mathrm{~g} /$ day intake $(2.58,1.44$ to $4.62, \mathrm{P}=0.003$ for trend) compared with zero intake. Results were consistent for Crohn's disease and ulcerative colitis (tests of heterogeneity: $\chi^{2} \mathrm{P}=0.17 ; \mathrm{I}^{2}=41 \%$ ). A similar pattern was observed for salty foods and snacks, where an increased risk of IBD was observed in participants with $\geq 100 \mathrm{~g} /$ day intake compared with $<50 \mathrm{~g} /$ day (2.06, 1.41 to 3.00, $\mathrm{P}<0.001$ for trend; supplementary table 8). Associations with similar direction were observed for the risk of Crohn's disease and ulcerative colitis, without significant heterogeneity $\left(\chi^{2} \mathrm{P}=0.25\right.$; $\left.\mathrm{I}^{2}=27.8 \%\right)$.

\section{Urinary sodium and risk of IBD}

Urinary sodium was used as a surrogate of dietary sodium intake. The risk of IBD in participants with higher levels of urinary sodium ( $\geq 3.5 \mathrm{~g} /$ day) was found not to be higher than in those patients with the lowest levels of urinary sodium $(<2.5 \mathrm{~g} /$ day, $\mathrm{P}=0.61$ for trend; table 4). As a large number of participants with IBD was found among the highest urinary sodium cohort, a sensitivity analysis was performed to further subdivide this cohort (supplementary table 9), but again no difference in risk of IBD was observed in those with the highest levels of urinary sodium $(\geq 5 \mathrm{~g} /$ day) compared with those with lower levels $(\mathrm{P}=0.45$ for trend). Although urinary sodium was found to be highest among participants from China (table 1) with only 19 incident cases of IBD, the results did not differ after exclusion of participants from China ( $\mathrm{P}=0.68$ for trend).

\section{Other food categories and risk of IBD}

Several other food categories were evaluated in exploratory analyses (table 5 and table 6, also see 


\begin{tabular}{|c|c|c|c|c|}
\hline & \multicolumn{4}{|c|}{ Ultra-processed food intake } \\
\hline & $\ll 1$ serving/day & $1-4$ servings/day & $\geq 5$ servings/day & $P$ trend \\
\hline \multicolumn{5}{|l|}{ Inflammatory bowel disease } \\
\hline No of participants & 76415 & 25453 & 11742 & \\
\hline No (\%) of events & $199(0.26)$ & $134(0.53)$ & $95(0.81)$ & \\
\hline Unadjusted model & 1 (ref) & $2.20(1.77$ to 2.74$)$ & $3.18(2.49$ to 4.07$)$ & $<0.001$ \\
\hline Minimally adjusted model $^{*}$ & 1 (ref) & 1.41 (1.11 to 1.79$)$ & $1.42(1.07$ to 1.90$)$ & 0.01 \\
\hline Fully adjusted modelt & 1 (ref) & 1.67 (1.18 to 2.37$)$ & $1.82(1.22$ to 2.72$)$ & 0.006 \\
\hline Fully adjusted plus AHEI score model & 1 (ref) & 1.75 (1.23 to 2.50$)$ & $1.92(1.28$ to 2.90$)$ & 0.004 \\
\hline Sensitivity analysis using multiple imputation $\neq$ & 1 (ref) & $1.54(1.21$ to 1.84$)$ & $1.71(1.22$ to 2.37$)$ & $<0.001$ \\
\hline \multicolumn{5}{|l|}{ Crohn's disease } \\
\hline No of participants & 76415 & 25453 & 11742 & \\
\hline No (\%) of events & $34(0.04)$ & $23(0.09)$ & $30(0.26)$ & \\
\hline Unadjusted model & 1 (ref) & 2.19 (1.29 to 3.72$)$ & $5.84(3.57$ to 9.54$)$ & $<0.001$ \\
\hline Minimally adjusted model ${ }^{*}$ & 1 (ref) & 1.15 (0.64 to 2.06$)$ & $1.92(1.05$ to 3.49$)$ & 0.07 \\
\hline Fully adjusted modelt & 1 (ref) & 2.72 (1.06 to 6.97$)$ & $4.50(1.67$ to 12.13$)$ & 0.01 \\
\hline Fully adjusted plus AHEI score model & 1 (ref) & $2.93(1.13$ to 7.60$)$ & $4.90(1.78$ to 13.45$)$ & 0.008 \\
\hline Sensitivity analysis using multiple imputation $\ddagger$ & 1 (ref) & $1.30(0.71$ to 2.37$)$ & $2.83(1.45$ to 4.65$)$ & 0.40 \\
\hline \multicolumn{5}{|l|}{ Ulcerative colitis } \\
\hline No of participants & 76415 & 25453 & 11742 & \\
\hline No (\%) of events & $165(0.22)$ & $111(0.44)$ & $65(0.55)$ & \\
\hline Unadjusted model & 1 (ref) & $2.20(1.73$ to 2.80$)$ & $2.63(1.97$ to 3.51$)$ & $<0.001$ \\
\hline Minimally adjusted model $^{*}$ & 1 (ref) & $1.48(1.13$ to 1.93$)$ & $1.27(0.91$ to 1.77$)$ & 0.02 \\
\hline Fully adjusted modelt & 1 (ref) & 1.55 (1.06 to 2.28$)$ & $1.46(0.93$ to 2.28$)$ & 0.08 \\
\hline Fully adjusted plus AHEI score model & 1 (ref) & 1.61 (1.09 to 2.38$)$ & $1.52(0.96$ to 2.41$)$ & 0.06 \\
\hline Sensitivity analysis using multiple imputation $\neq$ & 1 (ref) & 1.59 (1.23 to 1.98$)$ & 1.45 (0.96 to 2.12$)$ & $<0.001$ \\
\hline $\begin{array}{l}\text { AHEl=Alternate Healthy Eating Index. } \\
\text { Heterogeneity of results from Crohn's disease and ulc } \\
\text { ×Adjusted for age, sex, and geographical region. } \\
\text { †Adjusted for age, sex, geographical region, educatio } \\
\text { †To account for participants with missing data on foo }\end{array}$ & colitis: $x^{2} P=0.595$ & $\%$. & y intake, and location. & \\
\hline
\end{tabular}

supplementary tables 10-18 for detailed analyses). Intake of white meat, unprocessed red meat, dairy, starchy foods, and fruit, vegetables, and legumes was not associated with risk of IBD (supplementary tables 10-17). Intake of fried foods was associated with higher risk of IBD (supplementary table 18). Those with a fried food intake of $\geq 1$ servings/day showed the highest risk of $\operatorname{IBD}(3.02,1.51$ to $6.03, \mathrm{P}=0.006$ for trend) compared with those with zero intake. No significant difference in risk was observed between Crohn's disease and ulcerative colitis (tests of heterogeneity: $\chi^{2}$ $\mathrm{P}=0.19 ; \mathrm{I}^{2}=37.6 \%$ ).

\section{Discussion}

In this large, multinational, prospective cohort study involving 116087 participants from 21 low, middle, and high income countries, we found that higher intake of ultra-processed foods was associated with an increased risk of IBD. This was seen for all ultra-

\begin{tabular}{|c|c|c|c|c|}
\hline & \multicolumn{4}{|c|}{ Sodium intake (g/day) } \\
\hline & $<2.5$ & 2.5 to $<3.5$ & $\geq 3.5$ & P trend \\
\hline No of participants & 8444 & 11555 & 61033 & \\
\hline No (\%) of events & $42(0.50)$ & $73(0.62)$ & $269(0.44)$ & \\
\hline Unadjusted & 1 (ref) & $1.33(0.91$ to 1.95$)$ & 0.89 (0.64 to 1.23$)$ & 0.008 \\
\hline Minimally adjusted* & 1 (ref) & 1.88 (1.28 to 2.76$)$ & 1.66 (1.18 to 2.34$)$ & 0.004 \\
\hline Fully adjusted $t$ & 1 (ref) & 1.26 (0.80 to 1.97$)$ & $1.17(0.79$ to 1.74$)$ & 0.61 \\
\hline
\end{tabular}

processed foods, as well as individual types, including processed meats, soft drinks, refined sweetened foods, and salty foods and snacks. No significant heterogeneity was observed when the results for Crohn's disease were compared with the results for ulcerative colitis within each of these types of ultra-processed foods. These results remained consistent in sensitivity analyses using alternative classifications for ultra-processed food intake and using multiple imputation to account for missing FFQ data. The results were still consistent after adjustment for a western diet using the AHEI. Our findings support the hypothesis that intake of ultraprocessed foods could be an environmental factor that increases the risk of IBD.

\section{Comparison with other studies}

Processed foods are a diverse food group that include meats, dairy, starchy foods, fruit, vegetables, and legumes. None of these categories on its own was found to be implicated as a risk factor for IBD in our study. Recent attention has focused on diet as an environmental factor that might be implicated in the development of IBD, and studies have suggested that western-type diets that are typically high in protein, fat, salt, and sugar but low in fruit, vegetables, and fibre, are associated with increased risk of IBD. ${ }^{30-32}$ A recent meta-analysis suggested western-type diets were associated with a relative risk for IBD of 1.92 (95\% confidence interval 1.37 to 2.68$).{ }^{33}$ Western-type diets, however, also contain higher levels of additives and preservatives, which could explain the association 

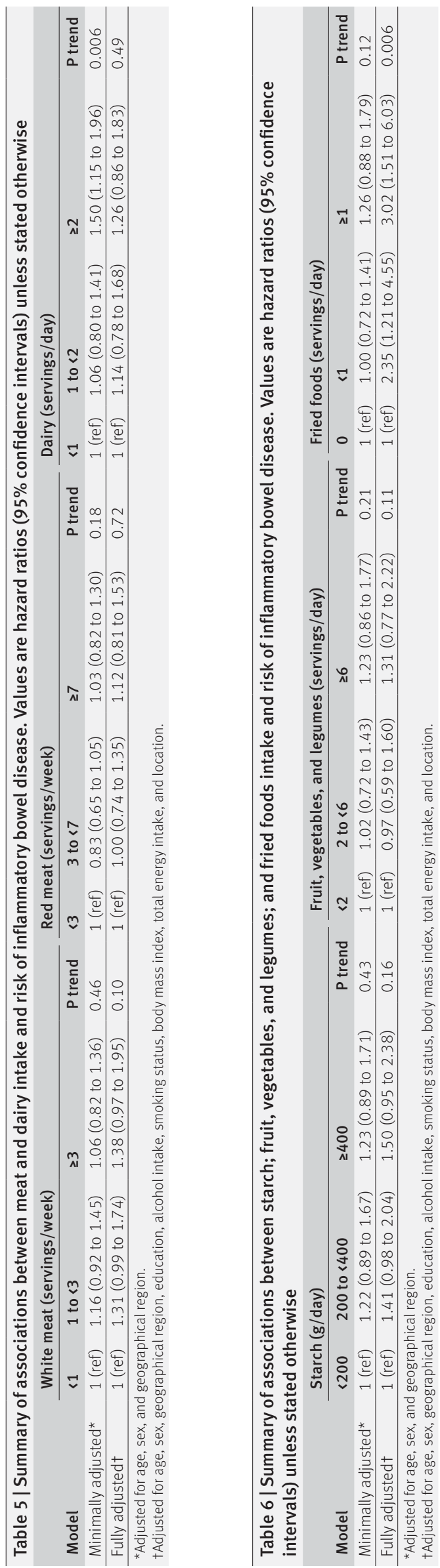

with higher risk of IBD. We hypothesised that increased sodium intake could be implicated, as various animal models have shown increased dietary sodium to be associated with exacerbation of autoimmune conditions, including collagen induced rheumatoid $\operatorname{arthritis}^{34}$ and 2,4,6-trinitrobenzenesulfonic acid induced colitis for IBD. ${ }^{35}$ In our study, however, we found no relation between urinary sodium, a surrogate for dietary sodium intake, and development of IBD. This suggests that components other than sodium in ultra-processed foods might be responsible for the higher risk of IBD observed with higher consumption of salty snacks.

Further studies are required to identify potential contributory factors in ultra-processed foods. Preclinical studies in mice models found that emulsifiers, which are widely used in western diets and include carboxymethylcellulose and polysorbate 80 , induce thinning of the mucosal layer and dysbiosis and promote development of colitis and colitis associated colon cancer. ${ }^{36-38}$ In preclinical studies other additives that have been implicated include maltodextrin 3940 and titanium dioxide. ${ }^{41}$ Intake of foods containing these types of additives could be a plausible pathway for disruption of gut microbiota and propagation of the subsequent immune activation that occurs in IBD.

Recent studies have assessed the association between processed food intake and IBD. One study from Romania and Belgium reported that participants with IBD were more likely to report higher intake of refined sweetened foods, processed and high fat meats, fried food, salt, ice cream, mayonnaise, margarine, and chips or other snacks compared with healthy controls. A US study using data from the National Health Interview Study in 2015 reported higher intake of cheese, cookies, French fries, sports drinks, and soda among participants with IBD compared with no IBD. These studies, however, had design limitations, including using a case-control or retrospective design, which are vulnerable to recall bias. A nested matched case-control study conducted using the large European Prospective Investigation into Cancer and Nutrition (EPIC) prospective database found that diets of high sugar and soft drink intake and low vegetable intake were associated with an increased risk of ulcerative colitis. ${ }^{5}$ A prospective study using data from the Nurses Health Study II cohort found that participants with a "prudent" dietary pattern in high school (higher intake of vegetables, fibre, and fish) showed a lower risk of subsequent Crohn's disease. ${ }^{42}$ A French study using the NutriNet-Sante prospective cohort did not find higher intake of ultra-processed foods among participants who subsequently developed IBD compared with those who did not, after adjustment for confounders, but this study might have been underpowered because of too few participants $(n=75)$ with IBD. ${ }^{15}$

We also found higher intake of fried food to be associated with higher risk of IBD. This association might exist because many fried foods are also processed (eg, chicken nuggets, French fries). It could be that the action of frying and the processing of oil leads to 
modification of nutrients within the food. ${ }^{4344}$ The type and quality of oil used might also be relevant. ${ }^{45}$

In our study, intake of white meat, unprocessed red meat, dairy, starch, and fruit, vegetables, and legumes were not found to be associated with risk of IBD. Recent meta-analyses that included high quality studies found no associations between pre-illness intake of carbohydrates, sugar, protein, or fat for either Crohn's disease or ulcerative colitis. ${ }^{46-48}$ Although some studies have suggested increased meat intake could increase the risk of IBD, ${ }^{1016}$ and higher fruit and vegetable intake might be protective, ${ }^{17}{ }^{18}$ these findings have not been found consistently in all studies that have examined this potential association. ${ }^{1049} 50$ This finding could be partly due to study design limitations, particularly in case-control studies where the selection of controls might have an impact on associations being evaluated.

For positive associations found in our study, similar directional effects were observed for Crohn's disease and ulcerative colitis. Overall, a lower number of participants with Crohn's disease were observed in our study $(n=90)$ compared with ulcerative colitis $(n=377)$. Reasons for the higher incidence of ulcerative colitis compared with Crohn's disease in our study probably reflects participants being from developing nations, where ulcerative colitis is known to be relatively more prevalent. ${ }^{51}$ Furthermore, the age of participants in the PURE study was 35-70 years, and the risk of Crohn's disease is lower at older ages, whereas ulcerative colitis has a bimodal peak, with many patients receiving a diagnosis in their 50s and 60s. ${ }^{152}$

\section{Strengths and limitations of this study}

We investigated dietary risk factors for IBD in a large prospective multinational cohort study. The study design overcomes the limitations of many of the existing studies in this specialty, which are retrospective or case-control in design and restricted to homogenous populations with limited external validity. ${ }^{10}$ The longitudinal design of our study allowed us to focus on people with incident IBD and to use medical record review and central adjudication to validate a sample of the diagnoses. Information on diet was collected at baseline before diagnosis to minimise risk of bias from reverse causation or recall bias. We excluded participants who received a diagnosis within one year of the baseline FFQ assessment to account for the possibility of dietary changes related to gastrointestinal symptoms that would later lead to a diagnosis of IBD. Validated, standardised, and country specific questionnaires were used for collection of dietary information. Because other baseline covariates on univariable analysis were found to be associated with risk of IBD, including smoking status and location, the validity of our study findings was increased. ${ }^{534} \mathrm{We}$ also identified other factors on univariable analysis, such as alcohol intake and BMI, that were significant but possibly correlated with other confounding factors and when put into a multivariable model were no longer associated with risk of IBD (analyses not shown).
Nonetheless, our study also has some potential limitations. Owing to the age of participants in the PURE study (35-70 years), incident Crohn's disease was observed in a relatively small number of participants, which might have resulted in our study being underpowered to determine risk factors for Crohn's disease. Furthermore, the generalisability of these findings to patients who develop IBD at an earlier age (children or young adulthood) is unclear. We did, however, run subgroup analyses to determine whether age was an effect modifier and found similar increased hazard ratios for risk of IBD in those younger than or older than 50 years. Only a sample of participants with IBD were selected for validation, which increases the risk of misclassification for cases that did not undergo verification processes, but misclassification of participants with IBD would tend to bias associations to the null and this should not affect our findings. We did not account for dietary changes over time, but dietary intake as measured by the FFQ has been shown to be relatively stable over time. ${ }^{55}$ During follow-up, however, some of the diets in included countries might have become partly westernised, which could lead to inaccuracy in capturing the degree of processed foods and their influence in these participants. FFQs are not an ideal instrument for measuring absolute intake-however, FFQs might still be useful to assess relative intake, so our results should be taken in the context of higher versus lower levels of ultra-processed food intake. ${ }^{56}$ Multiple comparisons were made and a possibility remains of positive results being found owing to chance alone. Our findings did, however, show a high level of statistical significance, thereby providing some reassurance that our observations are probably real. Lastly, although we were able to adjust for many variables through our multivariable models, owing to the observational nature of our study the possibility of residual bias from unmeasured (eg, antibiotic use in early childhood), imprecisely measured, or unknown confounders remains.

\section{Conclusions}

In this study, higher ultra-processed food intake was associated with higher risk of IBD. As white meat, unprocessed red meat, dairy, starch, and fruit, vegetables, and legumes were not found to be associated with development of IBD, this study suggests that it might not be the food itself that confers this risk but rather the way the food is processed or ultra-processed. A possibility also remains of residual confounding owing to unmeasured or unknown confounders. Further studies are needed to identify specific potential contributory factors among processed foods that might be responsible for the observed associations in our study.

\section{AUTHOR AFFILIATIONS}

'Department of Medicine (Division of Gastroenterology) and

Farncombe Family Digestive Health Research Institute, McMaster University, Hamilton, ON, Canada

${ }^{2}$ Population Health Research Institute, McMaster University and Hamilton Health Sciences, Hamilton, ON, Canada 
${ }^{3}$ Department of Internal Medicine, Universidad de La Frontera, Temuco, Chile

${ }^{4}$ Masira Research Institute, Universidad de Santander (UDES) Fundación Oftalmológica de Santander-FOSCAL-Bucaramanga, Colombia

${ }^{5}$ Department of Nutrition and Dietetics, Apollo Hospitals, Bangalore, India

${ }^{6}$ School of Public Health, Post Graduate Institute of Medical Education and Research, Chandigarh, India

${ }^{7}$ Achutha Menon Centre for Health Science Studies, SCTIMST and Health Action by People, Thiruvananthapuram, India

${ }^{8}$ Department of Cardiology, Estudios Clinicos Latinoamerica ECLA Rosario, Santa Fe, Argentina

${ }^{9}$ International Research Centre, Hospital Alemao Oswaldo Cruz, Sao Paulo, Brazil, Universidade Santo Amaro (UNISA), Sao Paulo, Brazil

${ }^{10}$ Department of Medicine, University of Ottawa, Ottawa, ON, Canada

${ }^{11}$ Faculté de pharmacie, Université Laval Institut universitaire de cardiologie et de pneumologie de Québec, Québec, Canada

${ }^{12}$ Department of Medicine, Division of Gastroenterology, College of Medicine, King Saud University, Riyadh, Saudi Arabia

${ }^{13}$ University of Health Sciences, Faculty of Medicine, Istanbul Sisli Hamidiye Etfal Health Training and Research Hospital, Clinic of Endocrinology and Metabolism Sisli/Istanbul, Turkey

${ }^{14}$ Faculty of Medicine and Defence Health, National Defence University of Malaysia, Kuala Lumpur, Malaysia

${ }^{15}$ Department of Physiology, University of Zimbabwe College of Health Sciences, Harare, Zimbabwe

${ }^{16}$ Department of Medicine, Queen's University, Kingston, ON, Canada

${ }^{17}$ School of Public Health, University of the Western Cape, Bellville. South Africa

${ }^{18}$ Institute for Community and Public Health, Birzeit University, Birzeit, Palestine

${ }^{19}$ Advocate Research Institute, Advocate Health Care, IL, USA

${ }^{20}$ School of Life Sciences, Independent University, Bangladesh Bashundhara, Dhaka, Bangladesh

${ }^{21}$ School of Public Health and Community Medicine, Institute of Medicine, Sahlgrenska Academy, University of Gothenburg, Göteborg, Sweden

${ }^{22}$ Department of Social Medicine, Wroclaw Medical University, Wroclaw, Poland

${ }^{23}$ Department of Community Health Sciences, The Aga Khan University, Karachi, Pakistan

${ }^{24}$ Medical Research and Biometrics Center, National Center for Cardiovascular Diseases, Fuwai Hospital, Chinese Academy of Medical Sciences Mentougou District, Beijing, China

${ }^{25}$ Section of Adult Medicine and Medical Research Unit, University of Philippines, Manila, Philippines

${ }^{26}$ Hatta Hospital, Dubai Medical University, Dubai Health Authority, Dubai, United Arab Emirates

${ }^{27}$ Isfahan Cardiovascular Research Center, Cardiovascular Research Institute, Isfahan University of Medical Sciences, Isfahan, Iran

${ }^{28}$ Department of Internal Medicine III, Division of Gastroenterology and Hepatology, Medical University of Vienna, Vienna, Austria We thank Shofiqul Islam for statistical advice and Dawn Agapay for help with the validation exercise.

Contributors: NN conceived and designed the study, compiled the data, did the statistical analysis, interpreted the data, and drafted the manuscript. ECLW compiled the data, did the statistical analysis, and drafted the manuscript. MD, AM, JM, and PM designed the study and drafted the manuscript. SR acquired the data and drafted the manuscript. FL, PLJ, PR, PVML, RPV, AO, AA, AW, PP, MAA, YA, KKN, IC, KY, TP, RK, RY, KBB, KZ, RI, LW, ZY, LS, AD, AY, and NM contributed data from their sites and reviewed the manuscript. WR conceived and designed the study and drafted the manuscript. SY conceived and designed the study, acquired the data and funding, and drafted the manuscript. NN is the guarantor. The corresponding author (NN), coauthors (EW, AM, MD, and SR), and senior author (SY) had full access to the data used for the study and made the final decision to submit the manuscript for publication. The corresponding author attests that all listed authors meet authorship criteria and that no others meeting the criteria have been omitted.
Funding: SY is supported by the Heart and Stroke Foundation and Marion W Burke chair in cardiovascular disease. The Prospective Urban Rural Epidemiology (PURE) study is an investigator initiated study funded by the Population Health Research Institute, Hamilton Health Sciences Research Institute, Canadian Institutes of Health Research (CIHR), Heart and Stroke Foundation of Ontario, support from CIHR's Strategy for Patient Oriented Research (SPOR) through the Ontario SPOR Support Unit, as well as the Ontario Ministry of Health and Long term Care and through unrestricted grants from several pharmaceutical companies, with major contributions from AstraZeneca (Canada), Sanofi-Aventis (France and Canada), Boehringer Ingelheim (Germany and Canada), Servier, and GlaxoSmithKline, and additional contributions from Novartis and King Pharma and from various national or local organisations in participating countries; these include: Argentina: Fundacion ECLA; Bangladesh: Independent University, Bangladesh and Mitra and Associates; Brazil: Unilever Health Institute, Brazil; Canada: Public Health Agency of Canada and Champlain Cardiovascular Disease Prevention Network; Chile: Universidad de la Frontera; China: National Center for Cardiovascular Diseases; Colombia: Colciencias (grant No 6566-04-18062); India: Indian Council of Medical Research; Malaysia: Ministry of Science, Technology and Innovation of Malaysia (grant Nos 100-IRDC/BIOTEK 16/6/21 (13/2007) and 07-05-IFN-BPH 010), Ministry of Higher Education of Malaysia (grant No 600-RMI/LRGS/5/3 (2/2011)), Universiti Teknologi MARA, Universiti Kebangsaan Malaysia (UKMHejim-Komuniti-15-2010); occupied Palestinian territory: the UN Relief and Works Agency for Palestine Refugees in the Near East, occupied Palestinian territory; International Development Research Centre, Canada; Philippines: Philippine Council for Health Research and Development; Poland: Polish Ministry of Science and Higher Education (grant No 290/W-PURE/2008/0), Wroclaw Medical University; Saudi Arabia: the Deanship of Scientific Research at King Saud University, Riyadh, Saudi Arabia (research group No RG-1436013); South Africa: the North-West University, SANPAD (South African and Netherlands programme for alternative development), National Research Foundation, Medical Research Council of South Africa, the South Africa Sugar Association (SASA), Faculty of Community and Health Sciences (UWC); Sweden: grants from the Swedish state under the Agreement concerning research and education of doctors; the Swedish Heart and Lung Foundation; the Swedish Research Council; the Swedish Council for Health, Working Life and Welfare, King Gustaf $V$ 's and Queen Victoria Freemasons Foundation, AFA Insurance, Swedish Council for Working Life and Social Research, Swedish Research Council for Environment, Agricultural Sciences and Spatial Planning, grant from the Swedish State under the Läkar Utbildnings Avtalet agreement, and grant from the Västra Götaland Region; Turkey: Metabolic Syndrome Society, AstraZeneca, Turkey, Sanofi Aventis, Turkey; United Arab Emirates: Sheikh Hamdan Bin Rashid Al Maktoum Award For Medical Sciences and Dubai Health Authority, Dubai, United Arab Emirates. The external funders of the PURE study had no input in the study design, data collection and analysis, interpretation, and preparation of the manuscript.

Competing interests: All authors have completed the ICMJE uniform disclosure form at www.icmje.org/coi_disclosure.pdf and declare: no support from the organisation listed in the funding statement for the submitted work; no financial relationships with any organisations that might have an interest in the submitted work in the previous three years, no other relationships or activities that could appear to have influenced the submitted work.

Ethical approval: This study was approved by the research ethics committees at all participating centres and by the Hamilton Integrated Research Ethics Board through protocol 03-206.

Data sharing: No additional data available.

The lead author (NN) affirms that the manuscript is an honest, accurate, and transparent account of the study being reported; that no important aspects of the study have been omitted; and that any discrepancies from the study as planned have been explained.

Dissemination to participants and related patient and public communities: Results from the PURE study are disseminated through press releases and social media.

Provenance and peer review: Not commissioned; externally peer reviewed.

This is an Open Access article distributed in accordance with the Creative Commons Attribution Non Commercial (CC BY-NC 4.0) license, which permits others to distribute, remix, adapt, build upon this work non-commercially, and license their derivative works on different terms, provided the original work is properly cited and the use is noncommercial. See: http://creativecommons.org/licenses/by-nc/4.0/. 
1 Loftus EVJr. Clinical epidemiology of inflammatory bowel disease: Incidence, prevalence, and environmental influences. Gastroenterology 2004;126:1504-17. doi:10.1053/i. gastro.2004.01.063

2 Maslowski KM, Mackay CR. Diet, gut microbiota and immune responses. Nat/mmunol 2011:12:5-9 doi:10.1038/ni0111-5

3 Lomer MC, Thompson RP, Powell J. Fine and ultrafine particles of the diet: influence on the mucosal immune response and association with Crohn's disease. Proc Nutr Soc 2002:61:123-30. doi:10.1079| PNS2001134

4 Thia KT, Loftus EVJr, Sandborn WJ, Yang SK. An update on the epidemiology of inflammatory bowel disease in Asia. Am J Gastroenterol 2008;103:3167-82. doi:10.1111/j.1572-0241.2008.02158x

5 Racine A, Carbonnel F, Chan SS, et al. Dietary Patterns and Risk of Inflammatory Bowel Disease in Europe: Results from the EPIC Study. Inflamm Bowel Dis 2016;22:345-54. doi:10.1097/ MIB.00000000000000638

6 Ananthakrishnan AN, Khalili H, Konijeti GG, et al. A prospective study of long-term intake of dietary fiber and risk of Crohn's disease and ulcerative colitis. Gastroenterology 2013;145:970-7. doi:10.1053/j. gastro.2013.07.050

7 Ananthakrishnan AN, Khalili H, Konijeti GG, et al. Long-term intake of dietary fat and risk of ulcerative colitis and Crohn's disease. Gut 2014;63:776-84. doi:10.1136/gutjnl-2013-305304

8 Cabré E, Domènech E. Impact of environmental and dietary factors on the course of inflammatory bowel disease. World / Gastroenterol 2012;18:3814-22. doi:10.3748/wjg.v18.i29.3814

9 Chapman-Kiddell CA, Davies PS, Gillen L, Radford-Smith GL. Role of diet in the development of inflammatory bowel disease. Inflamm Bowel Dis 2010;16:137-51. doi:10.1002/ibd.20968

10 Hou JK, Abraham B, El-Serag H. Dietary intake and risk of developing inflammatory bowel disease: a systematic review of the literature. Am Gastroenterol 2011:106:563-73 doi:10.1038/ajg.2011.44

11 de Silva PS, Olsen A, Christensen J, et al. An association between dietary arachidonic acid, measured in adipose tissue, and ulcerative colitis. Gastroenterology 2010;139:1912-7. doi:10.1053/i. gastro.2010.07.065

12 Hart AR, Luben R, Olsen A, et al. Diet in the aetiology of ulcerative colitis: a European prospective cohort study. Digestion 2008;77:5764. doi:10.1159/000121412

13 John S, Luben R, Shrestha SS, Welch A, Khaw KT, Hart AR. Dietary n-3 polyunsaturated fatty acids and the aetiology of ulcerative colitis: a UK prospective cohort study. Eur / Gastroenterol Hepatol 2010;22:602-6. doi:10.1097/MEG.0b013e3283352d05

14 Tjonneland A, Overvad K, Bergmann MM, et al, IBD in EPIC Study Investigators. Linoleic acid, a dietary n-6 polyunsaturated fatty acid, and the aetiology of ulcerative colitis: a nested case-control study within a European prospective cohort study. Gut 2009;58:1606-11. doi:10.1136/gut.2008.169078

15 Vasseur P, Dugelay E, Benamouzig R, et al. Dietary Patterns, Ultraprocessed Food, and the Risk of Inflammatory Bowel Diseases in the NutriNet-Santé Cohort. Inflamm Bowel Dis 2021;27:65-73. doi:10.1093/ibd/izaa018

16 Jantchou P, Morois S, Clavel-Chapelon F, Boutron-Ruault MC, Carbonnel F. Animal protein intake and risk of inflammatory bowel disease: The E3N prospective study. Am J Gastroenterol 2010;105:2195-201. doi:10.1038/ajg.2010.192

17 Li F, Liu X, Wang W, Zhang D. Consumption of vegetables and fruit and the risk of inflammatory bowel disease: a meta-analysis. Eur J Gastroenterol Hepatol 2015;27:623-30. doi:10.1097/ MEG.0000000000000330

18 Andersen V, Olsen A, Carbonnel F, Tjønneland A, Vogel U. Diet and risk of inflammatory bowel disease. Dig Liver Dis 2012:44:185-94. doi:10.1016/j.dld.2011.10.001

19 Swidsinski A, Ung V, Sydora BC, et al. Bacterial overgrowth and inflammation of small intestine after carboxymethylcellulose ingestion in genetically susceptible mice. Inflamm Bowel Dis 2009;15:359-64. doi:10.1002/ibd.20763

20 Roberts CL, Keita AV, Duncan SH, et al. Translocation of Crohn's disease Escherichia coli across M-cells: contrasting effects of soluble plant fibres and emulsifiers. Gut 2010;59:1331-9. doi:10.1136/ gut.2009.195370

21 Fiolet T, Srour B, Sellem et al $L$ Consumption of ultra-processed foods and cancer risk: results from NutriNet-Santé prospective cohort. BMJ 2018;360:k322. doi:10.1136/bmj.k322

22 Srour B, Fezeu LK, Kesse-Guyot E, et al. Ultra-processed food intake and risk of cardiovascular disease: prospective cohort study (NutriNet-Santé). BMJ 2019;365:11451. doi:10.1136/bmj.l1451

23 Dehghan M, Mente A, Rangarajan S, et al, Prospective Urban Rural Epidemiology (PURE) study investigators. Association of dairy intake with cardiovascular disease and mortality in 21 countries from five continents (PURE): a prospective cohort study. Lancet 2018:392:2288-97. doi:10.1016/S0140-6736(18)31812-9

24 Dehghan M, Mente A, Zhang X, et al, Prospective Urban Rural Epidemiology (PURE) study investigators. Associations of fats and carbohydrate intake with cardiovascular disease and mortality in 18 countries from five continents (PURE): a prospective cohort study. Lancet 2017:390:2050-62. doi:10.1016/S01406736(17)32252-3

25 Miller V, Mente A, Dehghan M, et al, Prospective Urban Rural Epidemiology (PURE) study investigators. Fruit, vegetable, and legume intake, and cardiovascular disease and deaths in 18 countries (PURE): a prospective cohort study. Lancet 2017:390:2037-49. doi:10.1016/S0140-6736(17)32253-5

26 Teo K, Lear S, Islam S, et al, PURE Investigators. Prevalence of a healthy lifestyle among individuals with cardiovascular disease in high-, middle- and low-income countries: The Prospective Urban Rural Epidemiology (PURE) study. JAMA 2013;309:1613-21. doi:10.1001/jama.2013.3519

27 Mente A, O'Donnell M, Rangarajan S, et al. Urinary sodium excretion, blood pressure, cardiovascular disease, and mortality: a community-level prospective epidemiological cohort study. Lancet 2018;392:496-506. doi:10.1016/S0140-6736(18)31376-X

28 Chiuve SE, Fung TT, Rimm EB, et al. Alternative dietary indices both strongly predict risk of chronic disease. J Nutr 2012;142:1009-18. doi:10.3945/jn.111.157222

29 Hiller L, Marshall A, Dunn J. Assessing violations of the proportional hazards assumption in Cox regression: does the chosen method matter?Trials 2015;16:134. doi:10.1186/1745-6215-16-S2-P134.

30 Albenberg LG, Lewis JD, Wu GD. Food and the gut microbiota in inflammatory bowel diseases: a critical connection. Curr Opin Gastroenterol 2012;28:314-20. doi:10.1097/ MOG.0b013e328354586f

31 Gentschew L, Ferguson LR. Role of nutrition and microbiota in susceptibility to inflammatory bowel diseases. Mol Nutr Food Res 2012;56:524-35. doi:10.1002/mnfr.201100630

32 Statovci D, Aguilera M, MacSharry J, Melgar S. The Impact of Western Diet and Nutrients on the Microbiota and Immune Response at Mucosal Interfaces. Front Immunol 2017;8:838. doi:10.3389/ fimmu. 2017.00838

33 Li T, Qiu Y, Yang HS, et al. Systematic review and meta-analysis: Association of a pre-illness Western dietary pattern with the risk of developing inflammatory bowel disease. J Dig Dis 2020;21:362-71. doi:10.1111/1751-2980.12910

34 Jung SM, Kim Y, Kim J, et al. Sodium Chloride Aggravates Arthritis via Th17 Polarization. Yonsei Med / 2019;60:88-97. doi:10.3349/ ymj.2019.60.1.88

35 Tubbs AL, Liu B, Rogers TD, Sartor RB, Miao EA. Dietary Salt Exacerbates Experimental Colitis. J Immunol 2017;199:1051-9. doi:10.4049/jimmunol.1700356

36 Chassaing B, Koren O, Goodrich JK, et al. Dietary emulsifiers impact the mouse gut microbiota promoting colitis and metabolic syndrome. Nature 2015;519:92-6. doi:10.1038/nature14232

37 Viennois E, Merlin D, Gewirtz AT, Chassaing B. Dietary EmulsifierInduced Low-Grade Inflammation Promotes Colon Carcinogenesis. Cancer Res 2017;77:27-40. doi:10.1158/0008-5472.CAN-161359

38 Chassaing B, Van de Wiele T, De Bodt J, Marzorati M, Gewirtz AT. Dietary emulsifiers directly alter human microbiota composition and gene expression ex vivo potentiating intestinal inflammation. Gut 2017:66:1414-27. doi:10.1136/gutinl-2016-313099

39 Laudisi F, Di Fusco D, Dinallo V, et al. The food additive maltodextrin promotes endoplasmic reticulum stress-driven mucus depletion and exacerbates intestinal inflammation. Cell Mol Gastroenterol Hepatol 2019:7:457-73. doi:10.1016/i.jemgh.2018.09.002

40 Nickerson KP, Chanin R, McDonald C. Deregulation of intestinal anti-microbial defense by the dietary additive, maltodextrin. Gut Microbes 2015:6:78-83. doi:10.1080/19490976.2015.1005477

41 Butler M, Boyle JJ, Powell J, Playford RJ, Ghosh S, et al. Dietary microparticles implicated in Crohn's disease can impair macrophage phagocytic activity and act as adjuvants in the presence of bacterial stimuli. Inflamm Res 2007:56:353-61. doi:10.1007/s00011-0077068-4

42 Ananthakrishnan AN, Khalili H, Song M; High School Diet and Risk of Crohn's Disease and Ulcerative Colitis. Inflamm Bowel Dis 2015;21:2311-9.

43 Jia B, Fan D, Li J, Duan Z, Fan L. Effect of Guar Gum with Sorbitol Coating on the Properties and Oil Absorption of French Fries. Int J Mol Sci 2017;18:2700. doi:10.3390/ijms18122700

44 Belkova B, Hradecky J, Hurkova K, Forstova V, Vaclavik L, Hajslova J. Impact of vacuum frying on quality of potato crisps and frying oil. Food Chem 2018:241:51-9. doi:10.1016/i.foodchem.2017.08.062

45 Chiou A, Kalogeropoulos N, Boskou G, Salta FN. Migration of health promoting microconstituents from frying vegetable oils to French fries. Food Chem 2012;133:1255-63. doi:10.1016/i. foodchem.2011.08.068

46 Zeng L, Hu S, Chen P, Wei W, Tan Y. Macronutrient Intake and Risk of Crohn's Disease: Systematic Review and Dose-Response Meta-Analysis of Epidemiological Studies. Nutrients 2017;9:500. doi:10.3390/nu9050500 
47 Wang F, Feng J, Gao Q, et al. Carbohydrate and protein intake and risk of ulcerative colitis: Systematic review and dose-response metaanalysis of epidemiological studies. Clin Nutr 2017:36:1259-65. doi:10.1016/j.clnu.2016.10.009

48 Wang F, Lin X, Zhao Q, Li J. Fat intake and risk of ulcerative colitis: Systematic review and dose-response meta-analysis of epidemiological studies. J Gastroenterol Hepatol 2017;32:19-27. doi:10.1111/jgh.13416

49 Reif S, Klein I, Lubin F, Farbstein M, Hallak A, Gilat T. Pre-illness dietary factors in inflammatory bowel disease. Gut 1997;40:754-60. doi:10.1136/gut.40.6.754

50 Halfvarson J, Jess T, Magnuson A, et al. Environmental factors in inflammatory bowel disease: a co-twin control study of a SwedishDanish twin population. Inflamm Bowel Dis 2006;12:925-33. doi:10.1097/01.mib.0000228998.29466.ac

$51 \mathrm{Ng} \mathrm{WK}$, Wong SH, Ng SC. Changing epidemiological trends of inflammatory bowel disease in Asia. Intest Res 2016;14:111-9. doi:10.5217/ir.2016.14.2.111

52 Cosnes J, Gower-Rousseau C, Seksik P, Cortot A. Epidemiology and natural history of inflammatory bowel diseases. Gastroenterology 2011;140:1785-94. doi:10.1053/j.gastro.2011.01.055
53 Mahid SS, Minor KS, Soto RE, Hornung CA, Galandiuk S. Smoking and inflammatory bowel disease: a meta-analysis. Mayo Clin Proc 2006;81:1462-71. doi:10.4065/81.11.1462

54 Benchimol El, Kaplan GG, Otley AR, et al. Rural and Urban Residence During Early Life is Associated with Risk of Inflammatory Bowel Disease: A Population-Based Inception and Birth Cohort Study. Am J Gastroenterol 2017;112:1412-22. doi:10.1038/ajg.2017.208

55 Nagel G, Zoller D, Ruf T, Rohrmann S, Linseisen J. Long-term reproducibility of a food-frequency questionnaire and dietary changes in the European Prospective Investigation into Cancer and Nutrition (EPIC)-Heidelberg cohort. Br J Nutr 2007;98:194-200. doi:10.1017/S0007114507691636

56 Steinemann N, Grize L, Ziesemer K, Kauf P, Probst-Hensch N, Brombach C. Relative validation of a food frequency questionnaire to estimate food intake in an adult population. Food Nutr Res 2017;61:1305193. doi:10.1080/16546628.2017.1305193

Supplementary information: appendices 1 and 2, tables 1-18, and figures 1 and 2 